SPICA Workshop, 03004 (2009)

DOI: $10.1051 /$ spica/200903004

(C) Owned by the authors, published by EDP Sciences, 2009

This is an Open Access article distributed under the terms of the Creative Commons Attribution-Noncommercial License, which permits unrestricted use, distribution, and reproduction in any noncommercial medium, provided the original work is properly cited.

\title{
DUSTY GIANTS GOING SAFARI
}

\author{
F. Kerschbaum, Th. Posch, and W. Nowotny
}

University of Vienna, Department of Astronomy, Türkenschanzstraße 17, A-1180 Wien, Austria

\begin{abstract}
With its high sensitivity and strongly reduced background, Safari-Spica allows for unprecedented studies of the mass loss processes in evolved stars, both from the compositional (mineralogical) and from the geometrical point of view. Apart from detailed galactic studies, for the first time also statistically meaningful samples of objects at different evolutionary phases in the Local Group galaxies with their wide spread of star formation histories as well as metallicities will be within reach.
\end{abstract}

Key words: Stars: AGB and post-AGB - Stars: mass-loss Galaxies: Local Group - Infrared: stars - Missions: SPICA

\section{INTRODUCTION}

The mid-infrared (MIR) and far-infrared (FIR) domains cover the critical wavelengths needed to characterize the physical and chemical processes that occur during the late stages of stellar evolution. These evolved red giants and Asymptotic Giant Branch (AGB) stars release most of their energy at infrared wavelengths. Key spectral diagnostics of the dust/ice composition, of the gas phase chemistry (e.g., water, HD, organic species) and of the prevailing physical conditions (atomic and ionic fine structure lines) cannot be observed with ground based telescopes, but are very well observable with the Safari instrument onboard the space telescope Spica (for technical details see the reviews by Nakagawa and Swinyard, this issue).

With its large field of view and flexible observing modes (broadband photometry, spectrophotometry or SED mode and spectroscopy with a resolution of typically 2000), Safari will reach much higher sensitivities when compared with previous FIR telescopes (IRAS, ISO, AKARI, Herschel). Safari will provide a superior way to obtain fully sampled spectroimages and SEDs of very faint and/or very extended regions through the gas and dust cycle in a wavelength domain not accessible to JWST or ALMA.

\section{Astromineralogy Legacy}

The principal objects injecting dust into the ambient ISM in our Galaxy are evolved stars; mainly red giants and Asymptotic Giant Branch (AGB) stars that represent the late evolutionary stages of solar type stars. A complex chemistry occurs in their cool extended stellar atmospheres $(\mathrm{T}=1000 \ldots 3000 \mathrm{~K})$. These outer layers are driven away from the star by radiation pressure and shocks waves from the photosphere, either episodically or by semicontinuous winds. Molecules easily form with the occuring species determined by the $\mathrm{C} / \mathrm{O}$ ratio. Some of these molecules aggregate onto dust grains, which under the action of radiation pressure accelerate and push the remaining gas, producing an expanding dusty molecular envelope. The circumstellar envelopes (CSEs) around AGB stars are very extended (typically a few AU!). Compared to millimeter and radio diagnostics that trace the external and colder CSE, only the FIR domain provides direct access to the inner, dense and warm circumstellar regions where the mass loss actually occurs and where dust grains form (amorphous and crystalline silicates, various oxides, hydrocarbon solids, $\mathrm{SiC}$, graphites, diamonds ....).

ISO caused a revolution in the field of astromineralogy of dusty environments from the earliest to the latest stages of stellar evolution (for an overview, see Henning 2003). Because of the relatively low sensitivity of the $60 \mathrm{~cm}$ telescope system, most of these findings were in the SWS(NIR/MIR)- and not in the LWS (FIR)-range. In the FIR high signal to noise spectra were possible only for high ML or very nearby sources. Spitzer, while having better sensitivity, was unfortunately not covering the whole infrared range, thus leaving significant gaps (see Fig. 1).

With Herschel, launched in May 2009, high sensitivity spectroscopy will be available, but starting only at $57 \mu \mathrm{m}$. Broad features - even in covered ranges - may be lost if located close to the respective edges of spectral sensitivity. What we therefore need is full cover, high sensitivity spectroscopy, and this will be provided by Safari.

\section{Astromineralogy potential}

\subsection{Some EXAMPLES}

The need for spectroscopy of cosmic dust over a wide wavelength range can be illustrated by several species of solids showing resonance features from the MIR to the FIR. Among them are refractory oxides such as hibonite (Mutschke et al., 2002), crystalline silicates such as forsterite (Bowey et al., 2002), hydrous silicates such as talc and montmorillonite (Mutschke et al., 2008), carbonates such 


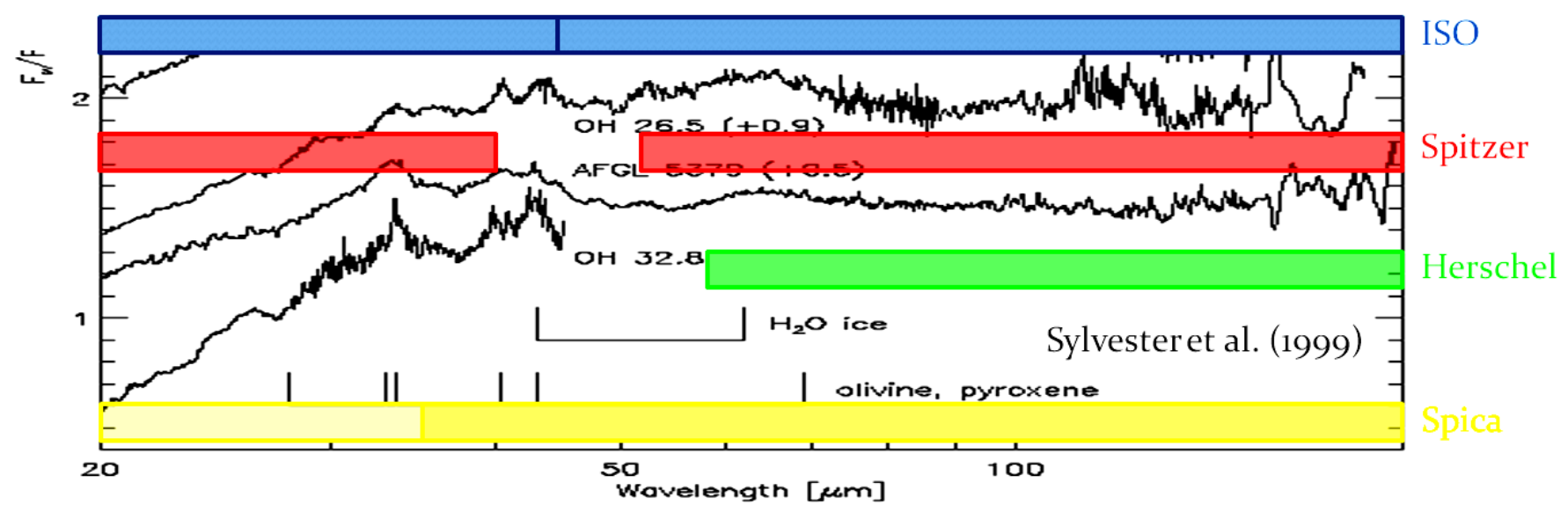

Figure 1. Spectroscopy ranges for some IR-missions in relation to ISO spectra taken from Sylvester et al. (1999).

as calcite and dolomite (Posch et al., 2007) as well as ices (e.g. Barlow, 1998). Some of these dust species may be not observable in the circumstellar dust of AGB stars, but rather in Young Stellar Objects or in Planetary Nebulae.

The general physical reason for solid state features spanning over a large spectral range is the simultaneous presence of different kinds of lattice vibrations. In hydrous silicates, for example, both inter-ionic vibrations such as Si-O stretching or bending modes and vibrations involving larger structures (such as sheets of ions) can be excited. While vibrations of lighter units cause spectral features at smaller wavelengths, vibrations of heavier units correspond to spectral features at larger wavelengths. Taken together, the different kinds of inter-ionic and inter-layer vibrations in hydrous silicates may lead to a pattern of infrared emission bands covering a whole decade $(10-100 \mu \mathrm{m})$ of wavelengths or more. The same is true for carbonates, e.g. calcite and dolomite, which exhibit several features between 7 and more than $100 \mu \mathrm{m}$ (for details cf. Posch et al., 2007). Amorphous water ice shows spectral features at $3.1,12.2$, and and 44-46 $\mu \mathrm{m}$ (Léger et al., 1983), while crystalline (hexagonal) water ice - prevailing at intermediate but not at very low temperatures - has an FIR feature at $62 \mu \mathrm{m}$ (Maldoni et al., 1999).

Table 1 lists a few mineral and ice species potentially occurring in the interstellar space, together with the peak positions of their emission features (where 'peaks' also include very broad structures in the respective spectra). It should be noted that most of these features have not yet been identified in the spectra of AGB stars or other dusty environments, partly due to a lack of high-sensitivity observational data obtained so far.

The need for spectroscopy of cosmic dust at high sensitivity becomes clear by considering the example of AGB stars in the globular cluster 47 Tuc. As shown by Lebzelter et al. (2006), the MIR spectra of the AGB stars in 47 Tuc can be used to trace the evolution of circumstellar dust in a homogeneous sample of giant stars and to correlate
Table 1. A selection of solid materials - minerals and ices - which show resonance features over a wide range of wavelengths. The values refer to spectra measured at or calculated for room temperature (300K). The last column denotes the particle shape to which the numbers refer: 'sph' for spherical grains, 'CDE' for a continuous distribution of ellipsoids, 'pow' for a powder composed of arbitrarily shaped grains. For references see the main text.

\begin{tabular}{lcc}
\hline Dust species & feature positions $[\mu \mathrm{m}]$ & shape \\
\hline Hibonite & $12.3,15.9,25.1,34.4,78.7$ & sph \\
Dolomite & $6.8,11.4,13.7,26.1$, & $\mathrm{CDE}$ \\
& $31.2,38.0,62.3$ & \\
Calcite & $7.0,11.4,14.0,30.7,43.7,90.1$ & $\mathrm{CDE}$ \\
Forsterite & $10.0,11.3,19.6,21.6,33.7$, & pow \\
& 49,69 & \\
Montmorillonite & $9.6,21.2,29.0,49.7,86.0,(105)$ & pow \\
Talc & $9.8,14.9,18.7,21.4,22.1$, & pow \\
& $25.4,28.9,32.0,38.7,43.2$, & \\
& $56.5,59.5,98.5$ & \\
Water ice & $3.1,12.2,44-46,62$ & pow \\
\hline
\end{tabular}

pulsational modes with dust shell properties. The Spitzer satellite made it possible for the first time to take detailled MIR spectra of stars as far away as the 47 Tuc stars (i.e., $5 \mathrm{kpc}$ ); however, even Spitzer's sensitivity proved insufficient at $20 \mu \mathrm{m}$ and beyond, where relevant dust features are located. Hence, circumstellar dust in globular clusters and external galaxies represent an ideal field of operation for Safari-Spica (see below).

\subsection{What SAFARI-SPICA CAN DO}

Taking the current sensitivity figures from Swinyard (this issue) and selecting two prototype AGB-stars, namely the O-rich Mira R Cas as a low/intermediate-MLR object and 
the IR-carbon star IRC+10216 for the High-MLR case one can derive some crude estimates what is possible on the field of dust mineralogy with Safari/Spica.

Based on its ISO SWS- and LWS-spectra low and intermediate-MLR objects can easily be studied out to $1 \mathrm{kpc}$. An observing time of 5 hours will provide a signal to noise ratio of more than 20 in the $30-100 \mu \mathrm{m}$ range.

Spectroscopy of High-MLR objects is possibly for targets as distant as the galactic centre. Again a guide number: IRC+10216, shifted outwards to a distance of $5 \mathrm{kpc}$, would require 3 hours integration time to reach a $S / N$ of 20 at $100 \mu \mathrm{m}$.

In addition to the mineralogical information, one may have 'for free' the highest transitions of e.g. coolants which are important in circumstellar envelopes - like $\mathrm{CO}$ and $\mathrm{H}_{2} \mathrm{O}$ for O-rich and $\mathrm{CO}$ and $\mathrm{HCN}$ in C-rich objects. These lines provide important information on the physical conditions of the innermost ML (driving) zones and are not accessable from the ground.

\section{Resolved Mass Loss History}

\subsection{Open Problems}

The evolution of mass loss over time as well as the geometrical changes are current key questions concerning the AGB mass loss process phenomenon. Increasing observational evidence supports a episodic AGB mass loss scenario, e.g. for CW Leo see Mauron \& Huggins (2006). Roughly spherical shells can be identified with separations corresponding to timescales of 200-800 years. Another, much longer, timescale for the variations in the mass-loss rate is expected from the thermal pulses which occur, depending on the core mass, typically on a time scale of about 10000 years. This is the interpretation of the interferometric observations of a detached shell around the star TT Cyg (Olofsson et al., 2000) observed in the CO(1-0) line. In the infrared, previous observations with IRAS have revealed extended shells, but even the better spatial resolution of ISOPHOT was clearly insufficient to really resolve the envelopes (e.g. Izumiura et al., 1996). Results from Spitzer and the AKARI Infrared Astronomy Satellite are of comparable quality. Clearly what is lacking at the moment are sensitive observations in the dust continuum over a larger FOV at a spatial resolution of a few arcsec. This would make it possible to trace details of the mass-loss history over a large timescale. There may be interrelations between the mass loss history and the change of geometry from the AGB- to the post-AGB phases.

Another interesting research area is the interaction region of the circumstellar envelope with the interstellar medium - a zone where one can expect both complex dynamics as well as chemistry. Recently a bow shock nebula at the interface between the stellar wind and the ISM was found for the first time around the AGB star R Hya (Ueta et al., 2006; Wareing et al., 2006). Such faint and low contrast phenomena need observations with high spatial resolution as well as high sensitivity/contrast in the far infrared.

\subsection{What Safari-Spica CAN DO}

After the pioneering results provided by ISO and Spitzer, Herschel (PACS and SPIRE) will observe spatially resolved nearby circumstellar envelopes survey for fossile shells in different environments, mainly already in the course of guaranteed time key projects. Safari-Spica will be able to build on these observations with its by orders of magnitude much higher sensitivity.

Taking again the instrumental parameters from Swinyard (this issue) and selecting now TT Cyg as the prototype object one can demonstrate Safari's potential in the field of probing the faint, extended emission of detached shells. For the nearest objects Safari will deliver detailed structures of detached envelopes, resolving timescales of much less than 1000 years, for TT Cyg, a prototype detached shell object at 500pc, 6" correspond to about 100 years!

Because of the well fitting field of view and the high sensitivity of Safari the more nearby ones (TT Cyg at $500 \mathrm{pc}$ ) can be imaged in only 0.5 hours! Even very short and low ML episodes will be detectable (including the expected faint ML modulations)

Going further out even at $1 \mathrm{kpc}$ the largest shells are still more or less filling the FOV and allow for a very efficient characterization of the mass loss history of the objects!

Since Safari will resolve bigger shells everywhere in the galaxy, one can expect quite a substantial gain in the statistics of these objects when analysing the foreseen large area surveys also in the view of evolved stars!

\section{MAss Loss in Clusters And EXternal Galaxies}

\subsection{SOME EXAMPLES}

The study of evolved red giants in stellar systems - within our galaxy or even beyond in extragalactic systems - will be an important and complementary topic for Safari-Spica. Compared to other missions, it offers a higher sensitivity in combination with an extended wavelength coverage (no gaps) as described in Sect. 2 and illustrated in Fig. 1. While the former is important for objects which are fainter at longer wavelengths (e.g. low mass loss rates), the latter will improve the mineralogical insights.

This will enable spectroscopic observations of AGB stars for example in galactic globular clusters as carried out for 47 Tuc with Spitzer by Lebzelter et al. (2006). The aim of such studies is to investigate the evolution of mass loss (mass loss rates, mineralogy of the dusty envelopes, etc.) along the AGB. Safari will improve this field especially for the more evolved objects $(>20 \mu \mathrm{m})$. 
An important add with respect to any other mission is the background limited sensitivity. Going to larger distances, i.e. for nearby extragalactic systems within the Local Group, Safari will be able to provide us with photometric data of point sources as exemplified in the next section. Colour-magnitude or colour-colour diagrams based on well chosen filters can be used to characterise the late-type stellar content of such systems as it was done in the past with IRAS (e.g. van der Veen \& Habing, 1988) or Spitzer (e.g. Blum et al., 2006; McQuinn et al., 2007; Jackson et al., 2007). Combined with data from earlier missions, this will allow to separate e.g. cold CSEs from e.g. molecular clouds.

Objects like TT Cyg which have quite low present mass loss rates but past episodes of strongly prononuced mass losses leading to detached shells of emitting cold dust (Olofsson et al., 2000) should be detectable with Safari even in the Magellanic clouds.

Although crowding may be a an issue for the imaging of more distant galaxies, the study of AGB stars in different environments will be interesting. The (relatively) large FOV of a few arcminutes will allow (quite expensive) mini-surveys in defined stellar populations (distance, metallicity, star formation history, etc.). Objects in external galaxies which were so far either studied only in the visual/NIR or were not detectable at all (high MLR) will be within reach even at the longest wavelengths of the IR range. In combination with other ground-/space-based data this will lead to a deeper understanding of the AGB mass loss process.

\subsection{What SAFARI-SPICA CAN DO}

Photometry of point sources is also a very fruitful and efficient domain of Safari. Taking the sensitivities from Swinyard (this issue) and putting our prototypes (R Cas, IRC+10216, as well as TT Cyg) at even larger distances the potential of our mission is evident.

Observations of AGB stars with intermediate mass loss rates turn out to be quite feasible out to the Magellanic Clouds (MC) with an R Cas type object needing only 1.5 hours for a $S / N$ of 20 ; at shortest wavelengths this would be much better but we take $100 \mu \mathrm{m}$ as the baseline again. AGB stars with high mass loss rates will be observable relatively easy out to the MCs in the full Safari range. Furthermore, a photometric detection of such objects will be feasible even in many of the (dwarf) galaxies of the Local group. As an example: IRC +10216 with an assumed distance of $200 \mathrm{kpc}$ would need only 0.5 hours of imaging time. The interesting cases of detached shell objects will be possible to be imaged at distances of the Magellanic clouds: TT Cyg at $50 \mathrm{kpc}$ will require exposure times of 1.5 hours for a $\mathrm{S} / \mathrm{N}$ of 20 at $100 \mu \mathrm{m}$.

\section{Conclusions}

With its broad and gapless wavelength coverage combined with high sensitivity Safari-Spica will allow deep insights into the physics of the inner parts of nearby CSEs covering dust and ice mineralogy, gas chemistry, and cooling.

The background limited sensitivity will reveal the detailed structure of resolved nearby CSEe probing both the mass loss history as well as the outflow/ISM interaction.

Large area, sensitive muti-use surveys in the galaxy but also in nearby local group systems will shed light on the mass loss process in different environments with e.g. different metallicity or star formation history.

\section{ACKNOWLEDGEMENTS}

This work has been supported by project P18939-N16 of the Austrian Science Fund (FWF) and and by project ASAP-CO016/03 of the Austrian Research Promotion Agency (FFG).

\section{REFERENCES}

Barlow, M.J. 1998, Ap\&SS, 255, 315

Blum, R.D., Mould, J.R., Olsen, K.A., et al. 2006, AJ, 132,2034

Bowey, J.E., Barlow, MJ., Molster, F.J., et al. 2002, MNRAS, 331, L1

Henning, Th. (ed.), Astromineralogy, Lecture Notes in Physics, Vol. 609, Springer

Izumiura, H., et al. 1996, A\&A, 315, L221

Jackson, D.C., Skillman, E.D., Gehrz, R.D., et al. 2007, ApJ, 667, 891

Lebzelter, Th., Posch, Th., Hinkle, K., Wood, P.R., \& Bouwman. J. 2006, ApJ, 653, L145

Léger, A., Gauthier, S., Defourneau, D., \& Rouan, D. 1983, A\&A, 117, 164

Maldoni, M. M., Robinson, G., Smith, R. G., Duley, W. W., \& Scott, A. 1999, MNRAS, 309, 325

Mauron, N., \& Huggins, P.J. 2006, A\&A, 452, 257

McQuinn, K.B.W., Woodward, Ch.E., Willner, S.P., et al. 2007, ApJ, 664, 850

Mutschke, H., Posch, Th., Fabian, D., \& Dorschner, J., 2002, A\&A, 392, 1047

Mutschke, H., Zeidler, S., Posch, Th., Kerschbaum, F., Baier, A., \& Henning, Th., 2008, A\&A 492, 117

Olofsson, H., et al. 2000, A\&A, 353, 583

Posch, Th., Kerschbaum, F., Richter, H., \& Mutschke, H., 2004, in: A. Wilson (ed.), The Dusty and Molecular Universe, ESA SP-577, 257

Posch, Th., Baier, A., Mutschke, H., \& Henning, Th., 2007, ApJ, 668, 993

Sylvester, R. J., Kemper, F., Barlow, M. J., et al. 1999, A\&A, 352, 587

Ueta T., et al. 2006, ApJ, 648, L39

Ueta, T. 2008, ApJ, 687, L33

van der Veen, W.E.C.J., \& Habing, H.J. 1988, A\&A, 194, 125

Wareing, C.J., et al. 2006, MNRAS, 372, L63 